\title{
Learning from our mistakes: We have a scientific and fiscal obligation to publish failed results
}

\author{
Carolyn M. Levinn* and Michael D. Pluth* \\ Department of Chemistry and Biochemistry, Materials Science Institute, Institute of Molecular Biology, \\ Knight Campus for Accelerating Scientific Impact. \\ University of Oregon, Eugene, OR 97403, USA \\ *Corresponding author E-mail: clevinn@uoregon.edu, pluth@uoregon.edu
}

\section{Summary:}

By only publishing the positive and successful results of our research, we as a field are limiting scientific advances and exercising poor stewardship of financial resources. By moving toward norms that include disseminating failed, unexpected, and tangential results, we have the opportunity to not only increase the efficiency of science, but also advance new discoveries. Of the broad scientific disciplines, chemistry in particular is poised to take advantage of already-existing, relatively low-barrier approaches to change the scientific publishing culture.

One of the great things about science is that it is supposed to be self-correcting by nature. Researchers and theorists publish their hypotheses, data, and interpretations, which form the basis for future iterations of experiments and analyses. These subsequent studies either support and further validate the earlier findings or provide new evidence contrary to initial interpretations. In many cases, such debates can live on in the literature for many years, often fueling the development of new methods to test experimental hypotheses that were as-of-yet inaccessible in earlier investigations. The more data that is available in these scenarios, even results that are inconclusive or seemingly uninteresting at the time, the more complete the analyses can be. From this perspective, all data are useful; all data are good data. Yet there remains a remarkable hesitation by researchers to publish null results or results that do not fit perfectly into the tidy package of a final manuscript.

This growing trend of only including positive data in published work is leading to a narrower and potentially less accurate view of science - cherry-picking only the data that agrees with what has been previously reported lends a clear bias to the research, which not only propagates inaccuracies, but also provides an incomplete picture of the real boundaries of new methods or ideas. ${ }^{1-3}$ Additionally, not making public what experiments did or did not work creates significant redundancy and waste: wasted time, wasted materials, and wasted money. Clearly some redundancy is important and serves as a key check in the literature, but this utility is lost if these experiments - both positive and negative - are not published in the first place. And while there is undoubtedly a benefit in researcher training to learn how to fail, reassess, and try something new, there is certainly room enough in science to accomplish this without wasting effort and mental health on experiments that others have already proven will fail. 
The growing reluctance to publish less interesting or null results comes at a cost. In 2016, the US federal government funded more than half (54\%) of the academic research and development efforts in the US. ${ }^{4}$ In total, this amounts to approximately $\$ 38.8$ billion dollars, which is about $1.1 \%$ of the estimated $\$ 3,561$ billion in tax revenue collected by the government that year. ${ }^{5}$ This is a lot of money (although arguably not enough), and the economic benefits to fundamental research are high and measurable. ${ }^{6,7}$ Without better information stewardship, however, we as a community are often inadvertently asking taxpayers to fund researchers to perform experiments that others have likely tried and know not to be successful. Pushing more information out to the scientific community helps to inform what techniques and methodologies work on what systems, establish and expand the limits of what can be done, and define key needs for future investigation. All of these aspects maximize efficiency, both in researcher training and scientific discovery.

We believe that this is especially tangible in chemistry. Using organic synthesis as an example, one can easily run hundreds of experiments or design and prepare novel compounds that end up not performing a desired function or are incompatible with later steps in a synthetic sequence. ${ }^{8}$ Such results are rarely published, or if they are may end up buried in the experimental section of an embargoed $\mathrm{PhD}$ thesis. Such compounds or reactions, however, help define the real substrate scope of new methodologies, displaying limitations or functional group tolerance on unique substrates that would otherwise not be tested. The characterization of intermediates, whether or not useful to the original story, can guide future researchers in trying to decipher what they themselves have prepared. Such unwanted or uninteresting compounds may prove to be of high value in the future in areas of chemistry that have yet to be discovered and expanded. In essence, future researchers may want to make a compound that has already been prepared but was never published because it did not fit cleanly into an overarching narrative. By not making this information available and accessible, we are in many cases hindering the field, slowing progress, and wasting time and other resources in the scientific community, as well as the limited taxpayer money. Put simply, we have an obligation to publish scientifically sound, "failed" results, not just ones packaged in a nice, neat success story.

This principle of increasing the dissemination of all data, useful or not, gained some traction in the early 2010's with a number of publishing platforms establishing entire journals designated for publishing negative or null results. ${ }^{9,10}$ Such efforts, however, have met limited success. Many of the journals folded within a few years, due in part to the fact that publishing null results is not incentivized by most academic structures, such as impact factors, citation rates, or journal prestige. ${ }^{2,11}$

Learning from these failed publishing experiments, the question remains: how can we, the creators and consumers of scientific information and outcomes, ensure that this information is available? Is there a place in the scientific literature for unsuccessful experiments and failed results? Some might argue that no including failed experiments would decrease general readability of the literature, exploding a four page 
communication into a 300 page saga. Or, one might contend that failed results often lead to spin-off projects within the same labs, and that disseminating that information puts researchers, especially those early in their careers, at risk of being scooped by more established competing labs that have more resources.

One potential solution could involve publishing on open-access multi-component platforms, such as Octopus, currently under development by a team led by Dr. Alexandra Freeman. ${ }^{12-14}$ This approach rejects the classical idea of publishing a paper or article as a 'unit' of science - instead favoring a system where any researcher can publish a result or an idea as a piece in the chain of research, rather than as a completed story. Such platforms offer many more opportunities for often overlooked or insignificant results, both negative and positive, to be made public.

Another solution could be requiring that any federally funded research projects publish their results, both negative and positive, within a set time frame of the end of the grant period. Such a system would provide a time buffer for labs to develop spin-off projects from unanticipated findings, but still ensures that all information is eventually made public, hopefully minimizing redundancy without reward.

More broadly, this is a conversation that needs to be had, with potential solutions and paths forward discussed and debated at length. While there is no one perfect solution right now, increasing visibility of the problem can help, and, there are some relatively straight-forward ways that people can start. As an open access platform, most supplemental information (SI) files are available even without subscriptions to the specific journal. A starting solution could be including a second SI file, or even just a subsection in the normal SI, that includes experiments that were tried and unsuccessful, or that were successful but did not go anywhere or were not pursued. In fact, some researchers are already including similar sections in their SIs. ${ }^{15,16}$ One major limitation of this solution is that such files are not generally indexed, which makes broad capture of the information less likely, however this approach would put the results where at least researchers in that field who would likely benefit the most might be most likely to find it. This certainly would look different from field to field, and of course is more tractable in some areas than others. However, in some areas, such as synthetic organic chemistry, the obvious barriers are not so high, and this would be a good place to start.

We are confident that even small steps toward this larger goal can provide a real impact, both with regard to scientific rigor and economic sensibility. We have a duty to the taxpayers to maximize our efficiency as best as possible, while maintaining high research standards. We view that the field of chemistry is poised to play a leadership role in this endeavor, and challenge chemists to think about new ways of publishing the full results of their work. Our lab will be making an effort to include things that 'don't work' in the SIs of future papers as well as in other outlets starting in 2020, and we invite other research groups to join us in this effort. 
This work was supported, in part by the NSF (CML: DGE-1309047; MDP: CHE-1506221),

\section{References}

1. Franco, A.; Malhotra, N.; Simonovits, G. Publication bias in the social sciences: Unlocking the file drawer. Science 2014, 345, 1502-1505.

2. The All Results Journals: Chem. http://arjournals.com/index.php/Chem/index (January 212020 )

3. Kluger, J. Why Scientists Should Celebrate Failed Experiments. TIME, 2014, https://time.com/3206754/publication-bias-null-results/

4. National Science Board. 2018. Science and Engineering Indicators 2018. NSB-2018-1. Alexandria, VA: National Science Foundation. Available at https://www.nsf.gov/statistics/indicators/

5. Federal Tax Revenue by Source, 1934 - 2018. https://taxfoundation.org/federal-tax-revenuesource-1934-2018/ (January 21 2020)

6. Papadakis, M. The economic impacts of public science can be measured. Scientist 1997, 11, 8-8.

7. Salter, A. J.; Martin, B. R. The economic benefits of publicly funded basic research: a critical review. Research Policy 2001, 30, 509-532.

8. Sierra, M. A.; de la Torre, M. C. Dead ends and detours en route to total syntheses of the 1990s. Angewandte Chemie-International Edition 2000, 39, 1538-1559.

9. Publishing Failure in Science, Martin, K., https://www.goldbio.com/articles/article/PublishingFailure-in-Science, (January 212020 )

10. Hilten, L. G. v. Why it's time to publish research "failures". Elsevier Connect, 2015, https://www.elsevier.com/connect/scientists-we-want-your-negative-results-too

11. New Negatives in Plant Science. https://www.journals.elsevier.com/new-negatives-in-plantscience

12. Dr Alexandra Freeman - 'Octopus: a radical new approach to scientific publishing'. https://royalsociety.org/topics-policy/projects/research-culture/research-culture-conference/thepitch/the-pitch-winner-dr-alexandra-freeman/ (January 21 2020)

13. Freeman, A. Innovator Story: Wrestling the Octopus. eLife Innovation, 2019, https://elifesciences.org/labs/9fdd6f3c/innovator-story-wrestling-the-octopus

14. Octopus - All of Science in One Place. https://octopus-hypothesis.netlify.com/ (January 21 2020)

15. Park, H.; Verma, P.; Hong, K.; Yu, J. Q. Controlling Pd(IV) reductive elimination pathways enables $\mathrm{Pd}(\mathrm{II})$-catalysed enantioselective $\mathrm{C}\left(\mathrm{sp}^{3}\right)$-H fluorination. Nature Chemistry 2018, 10, 755-762.

16. Eshon, J.; Foarta, F.; Landis, C. R.; Schomaker, J. M. alpha-Tetrasubstituted Aldehydes through Electronic and Strain-Controlled Branch-Selective Stereoselective Hydroformylation. Journal of Organic Chemistry 2018, 83, 10207-10220. 